Marquette University

e-Publications@Marquette

Biomedical Engineering Faculty Research and

Publications

Biomedical Engineering, Department of

$9-1-2018$

\title{
Technical Note: Enhancing Soft Tissue Contrast And Radiation-Induced Image Changes With Dual-Energy CT For Radiation Therapy
}

\author{
George Noid \\ Medical College of Wisconsin \\ An Tai \\ Medical College of Wisconsin \\ Diane Schott \\ Medical College of Wisconsin \\ Nilesh Mistry \\ Siemens Medical Solutions USA, Inc \\ Yu Liu \\ Medical College of Wisconsin
}

See next page for additional authors

Follow this and additional works at: https://epublications.marquette.edu/bioengin_fac

Part of the Biomedical Engineering and Bioengineering Commons

\section{Recommended Citation}

Noid, George; Tai, An; Schott, Diane; Mistry, Nilesh; Liu, Yu; Schmidt, Taly Gilat; Robbins, Jared R.; and Li, X. Allen, "Technical Note: Enhancing Soft Tissue Contrast And Radiation-Induced Image Changes With Dual-Energy CT For Radiation Therapy" (2018). Biomedical Engineering Faculty Research and Publications. 599.

https://epublications.marquette.edu/bioengin_fac/599 


\section{Authors}

George Noid, An Tai, Diane Schott, Nilesh Mistry, Yu Liu, Taly Gilat Schmidt, Jared R. Robbins, and X. Allen $\mathrm{Li}$

This article is available at e-Publications@Marquette: https://epublications.marquette.edu/bioengin_fac/599 
Marquette University

e-Publications@Marquette

\section{Biomedical Engineering Faculty Research and Publications/College of} Engineering

This paper is NOT THE PUBLISHED VERSION; but the author's final, peer-reviewed manuscript. The published version may be accessed by following the link in the citation below.

Medical Physics, Vol. 45, No. 9 (September 2018): 4238-4245. DOI. This article is (C) American Association of Physicists in Medicine and permission has been granted for this version to appear in $\underline{\mathrm{e}}-$ Publications@Marquette. American Association of Physicists in Medicine does not grant permission for this article to be further copied/distributed or hosted elsewhere without the express permission from American Association of Physicists in Medicine.

\section{Technical Note: Enhancing Soft Tissue Contrast And Radiation-Induced Image Changes With Dual-Energy CT For Radiation Therapy}

George Noid

Department of Radiation Oncology, Medical College of Wisconsin, Milwaukee, WI

An Tai

Department of Radiation Oncology, Medical College of Wisconsin, Milwaukee, WI

Diane Schott

Department of Radiation Oncology, Medical College of Wisconsin, Milwaukee, WI Nilesh Mistry

Siemens Medical Solutions USA, Inc., Malvern, PA

Yu Liu

Department of Radiology, Medical College of Wisconsin, 8701 Watertown Plank Rd., Milwaukee, WI 


\section{Taly Gilat-Schmidt}

Department of Biomedical Engineering, Marquette University, Milwaukee, WI

Jared R. Robbins

Department of Radiation Oncology, Medical College of Wisconsin, Milwaukee, WI

X. Allen Li

Department of Radiation Oncology, Medical College of Wisconsin, Milwaukee, WI

\section{Abstract \\ Purpose}

The purpose of this work is to investigate the use of low-energy monoenergetic decompositions obtained from dual-energy CT (DECT) to enhance image contrast and the detection of radiation-induced changes of CT textures in pancreatic cancer.

\section{Methods}

The DECT data acquired for 10 consecutive pancreatic cancer patients during routine nongated CT-guided radiation therapy (RT) using an in-room CT (Definition AS Open, Siemens Healthcare, Malvern, PA) were analyzed. With a sequential DE protocol, the scanner rapidly performs two helical acquisitions, the first at a tube voltage of $80 \mathrm{kVp}$ and the second at a tube voltage of $140 \mathrm{kVp}$. Virtual monoenergetic images across a range of energies from 40 to $140 \mathrm{keV}$ were reconstructed using an image-based material decomposition. Intravenous (IV) bolus-free contrast enhancement in pancreas patient tumors was measured across a spectrum of monoenergies. For treatment response assessment, the changes in CT histogram features (including mean CT number (MCTN), entropy, kurtosis) in pancreas tumors were measured during treatment. The results from the monoenergetic decompositions were compared to those obtained from the standard $120 \mathrm{kVp}$ CT protocol for the same subjects.

\section{Results}

Data of monoenergetic decompositions of the 10 patients confirmed the expected enhancement of soft tissue contrast as the energy is decreased. The changes in the selected CT histogram features in the pancreas during RT delivery were amplified with the low-energy monoenergetic decompositions, as compared to the changes measured from the $120 \mathrm{kVp}$ CTs. For the patients studied, the average reduction in the MCTN in pancreas from the first to the last (the 28th) treatment fraction was $4.09 \mathrm{HU}$ for the standard $120 \mathrm{kVp}$ and $11.15 \mathrm{HU}$ for the 40 keV monoenergetic decomposition.

\section{Conclusions}

Low-energy monoenergetic decompositions from DECT substantially increase soft tissue contrast and increase the magnitude of radiation-induced changes in CT histogram textures during RT delivery for pancreatic cancer. Therefore, quantitative DECT may assist the detection of early RT response.

\section{Introduction}

\section{A. Theory}

It is highly desirable to improve CT soft tissue contrast for radiation therapy (RT). The delineation of targets and organs at risk (OAR) for treatment planning and delivery guidance could be improved by enhanced soft tissue contrast. Furthermore, increased soft tissue contrast could potentially increase the sensitivity of CT for treatment response. Contrast in CT depends on differences in the linear attenuation coefficient of the tissue 
types being scanned, a quantity that is a function of x-ray energy, electron density, and atomic composition. Generally speaking, the lower the energy of the $x$ ray, the larger the soft tissue contrast.

Dual-Energy CT (DECT) is an emerging imaging technology in radiation oncology that utilizes the combination of two different $x$-ray spectra during image acquisition.1-3 Many approaches have been developed over the years, including systems that use rapid voltage switching, two sources, layered detectors, splitting the x-ray beam at the source (using filtration), or acquiring images sequentially at different $k V$ settings. Postprocessing of the spectral information provides a wealth of clinically relevant information. The information can be used to reconstruct virtual monoenergetic images that emphasize differences in material especially at lower keV values. $\underline{1}$

The virtual monoenergetic decomposition of DECT was described as early as 1976.2 The method typically depends on basis material decomposition and the knowledge of the mass attenuation of the basis materials. $\underline{3}$ Virtual monoenergetic images (MEI) resemble a CT acquired at a single energy. Standard polyenergetic $\mathrm{CT}$ images (PEI) are reconstructed from an x-ray spectrum typically defined by the tube potential (i.e., $120 \mathrm{kVp}$ ). These images average over the energy-dependent differences in the linear attenuation. MEI can enhance the image quality by exploiting the enhancement in contrast at x-ray energies below $70 \mathrm{keV}$.

The energy dependence of the linear attenuation coefficients for monoenergetic $\mathrm{x}$ rays can be calculated from formulae derived in a publication by Watanabe. $\underline{4}$ The CT number (CTN) of a material in Hounsfield units (HU) and the contrast are then calculated from the following formula where the linear attenuation coefficient, $\mu$, is a function of x-ray energy:

$$
\begin{aligned}
& C T N=1000\left(\frac{\mu-\mu_{\text {water }}}{\mu_{\text {water }}-\mu_{\text {air }}}\right) \\
& C T N=1000\left(\frac{\mu-\mu_{\text {water }}}{\mu_{\text {water }}-\mu_{\text {air }}}\right)(1) \\
& \text { Contrast }=\left|\overline{C T N_{A}}-\overline{C T N_{B}}\right| \\
& \text { Contrast }=\left|\overline{C T N_{A}}-\overline{C T N}_{B}\right|(2)
\end{aligned}
$$

Thus, we can calculate the expected CT contrast between materials $A$ and $B$, as a function of $\mathrm{x}$-ray energy. In Eq. 2, $\overline{C T N}_{A}$ is the average CT number in a given uniform region of interest of material $A$. CT contrast is at a maximum at the minimum achievable monoenergy, which is $40 \mathrm{keV}$ for the DECT protocol applied. However, it is known that noise also increases as the monoenergy decreases. $\underline{\mathbf{5}}$

\section{B. Applications}

The MEIs of DECT are the subject of numerous studies in diagnostic radiology, which typically use IV bolus. Yu et al. reviewed clinical applications for beam-hardening corrections, contrast and noise optimization, metal artifact reduction, and material differentiation. $\underline{\mathbf{3}}$ Schneider et al. explored the enhanced contrast to noise ratio (CNR) and vessel attenuation of cervical and cerebral DECT angiographic studies. 6 Wichmann et al. reported an enhanced CNR, subjective image quality, and tumor delineation of head and neck squamous cell carcinoma.7 Sudarski et al. evaluated low-energy MEI for enhanced CNR in abdominal and lower extremity angiography. $\underline{\mathbf{8}}$ The following year Sudarski et al. evaluated subjective and objective image quality enhancements for MEl of gastrointestinal stromal tumors. $\underline{9}$ Kaup et al. found the optimal MEl energy level for subjective and objective image quality of lung cancer lesions.10 Two studies (Frellessen et al. and Hardie et al.) demonstrated a contrast enhancement for diagnostic pancreatic imaging.11, 12 Furthermore, it has been shown that enhancements in CT image quality reduce uncertainties in patient positioning during image-guided radiation 
therapy (IGRT) and hence can reduce setup margins during treatment planning.13 It follows that contrast enhancement from DECT would improve the quality of IGRT planning and delivery.

Beyond simply improving the image quality, low-energy MEI could potentially improve the use of CT for treatment response assessment due to its superior sensitivity to the change in material composition. It has been reported that radiation can induce changes in $\mathrm{CT}$ texture features during RT of several cancers including pancreas, head and neck, lung, and liver.14-17 Quantitative data extracted from daily CTs acquired during IGRT using in-room CT, such as changes in CT textures (histogram or higher order features) may be used to assess radiation treatment response.14 However, CT-based detection of the response depends on the image quality (contrast, noise, spatial resolution). Lesions embedded in soft tissue, such as the pancreas, are difficult to detect with $\mathrm{CT}$ because the soft tissue contrast is inadequate and hence the treatment response signal is relatively small. On average a change in 4.7 HU on MCTN in the pancreas is reported between the first week and last week of therapy based on conventional $120 \mathrm{kVp}$ CTs, which is almost 10 times larger than the standard deviation of the MCTN of water for this scanner.14 In comparison to traditional PEI CTs, we investigated how low x-ray energy MEI CT enhances the soft tissue contrast and thus amplifies the signal of radiation-induced changes in CT textures.

In this study, we first quantified the contrast enhancement of MEI/DECT based on patient data with pancreatic cancers for low and high x-ray monoenergies. Second, we investigated the enhancement of MEI/DECT on the detection of treatment response during RT for pancreatic cancer based on quantitative analysis of daily DECTs.

\section{Materials and methods}

\section{A. Hardware components}

All CT data were acquired on a 64-slice CT scanner (SOMATOM Definition AS Open; Siemens Medical Solutions USA, Inc., Malvern, PA). The scanner was installed in a linac room on a sliding gantry (CT-on-rails). The standard PEI scans were performed at a tube potential of $120 \mathrm{kVp}$, a pitch of 0.6 , tube rotation time of $0.5 \mathrm{~s}$, volume computed CT dose index (CTDIvol) of $16.99 \mathrm{mGy}$, and a scan resolution of $0.98 \times 0.98 \mathrm{~mm}$ in the scanning plane with a 3-mm slice thickness. Scan times vary from day to day and patient to patient based on the specific scan length chosen from each day's topogram. The raw data were reconstructed using a medium sharp convolution kernel (30f).

The DECT data were acquired on the same scanner using a sequential protocol known as Dual Spiral Dual Energy. The protocol generated two CT datasets through successive helical scans at different $\mathrm{kV}$ and $\mathrm{mA}$ levels (but the same tube rotation time, $0.5 \mathrm{~s}$ ). The first and the second scan were at $80 \mathrm{kVp}$ and $120 \mathrm{kVp}$ with a pitch of 0.6 and 1.2 , respectively. The scan resolution was $0.98 \times 0.98 \mathrm{~mm}$ with a $3-\mathrm{mm}$ slice thickness, equal to that for the PEI. CARE Dose 4D (Siemens Healthcare) was used to reduce the dose to the patient. CARE Dose is an automatic exposure-control algorithm that modulates the tube current in the angular and longitudinal direction based on the patient girth as measured in a topogram. $\underline{\mathbf{1 8}}$ Thus, the dose to the patients in the study varied but the combined reference dose level was set equal to a standard abdominal CT (16.99 mGy). The reference dose or "Reference $\mathrm{mAs}$ " is a parameter entered by the user to determine the desired level of image quality. It is an effective $m A s$ that produces the desired image quality on a standard sized patient. MEI reconstructions were performed at the scanner using the built in MEl image-based algorithm from 40 to $140 \mathrm{keV}$ with a medium sharp convolution kernel (30f) and an iterative reconstruction (SAFIRE strength 5; Siemens Healthcare) to reduce noise, which increases as MEI energy decreases. $\underline{\mathbf{5}}$ 19 SAFIRE does not alter image contrast as has been previously demonstrated on patient and phantom scans. $\underline{\mathbf{2 0}}$ 


\section{B. Clinical data}

The pancreatic cancer patients selected in this study were treated with preoperative RT of 50.4 Gy in 28 daily fractions. The daily noncontrast standard CT or DECT was acquired at alternative fractions during the 6-week RT duration as a part of regular IGRT using the CT-on-rails system. The data for 10 consecutive patients with respiration motion of pancreas head (as measured from a 4DCT acquired at simulation) less than $7 \mathrm{~mm}$ were analyzed. The 7-mm criterion is necessary because the sequential DECT protocol is not compatible with respiration gating. For patients with more than $7 \mathrm{~mm}$ of target motion, gated IGRT is the standard of care in our clinic. The DECT was scanned with the Dual Spiral Dual Energy protocol. MEls were generated across a range of $x$-ray energies for comparison to the standard PEIs. In both image types, the targets and OAR were contoured manually. If a stent was present in the bile duct for a pancreatic cancer case, it was delineated and excluded from the analysis. Imaging contrast defined as the difference in CTN between the targets (pancreas head) and OAR (duodenum) in both MEls and PEIs acquired during consecutive IGRT fractions [see Eq. 2] was assessed from the patient data.

In addition, the daily $\mathrm{CT}$ and $\mathrm{DECT}$ data were quantitatively analyzed to detect radiation-induced changes of $\mathrm{CT}$ histogram features in the target volume (e.g., pancreatic head). Treatment responses as evaluated through changes in CT textures of the target for the MEls and PEls were compared. The change in MCTN of the aorta was also assessed in these images in order to establish CTN stability. All contours were manually drawn by a single physicist and checked with a radiation oncologist using a commercial tool (MIM version 6.4.5 (Build F204-00), MIM Software Inc., Cleveland, $\mathrm{OH}$ ). The changes in the selected histogram features (MCTN, skewness, kurtosis, entropy, standard deviation) were calculated for all 10 patients using an in-house program developed in MATLAB (version 2012b; MathWorks, Natick, MA). The histogram features evaluated are defined in the MATLAB Statistics and Machine Learning Toolbox.

\section{Results}

From the selected patient data, we calculated the contrast [Eq. 2] between the pancreas head and the duodenum which is shown in Fig. 1 (a). The average contrast increases from $10.4 \pm 7.9$ to $24.6 \pm 16.2 \mathrm{HU}$ when comparing the data from the $120 \mathrm{kVp}$ images to those for the $40 \mathrm{keV}$ images. This is an increase of $189 \%$. The reported uncertainty is the standard deviation of the calculated contrasts for all 10 patients. In Fig. 1, (b) and (c) are axial slices from one of the selected patients [\#9 in Fig. 1(a)] demonstrating the contrast enhancement when comparing a $120 \mathrm{kVp} \mathrm{PEI}$ and a $40 \mathrm{keV}$ MEI.

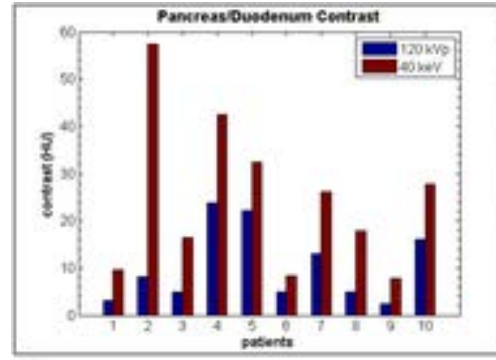

(a)

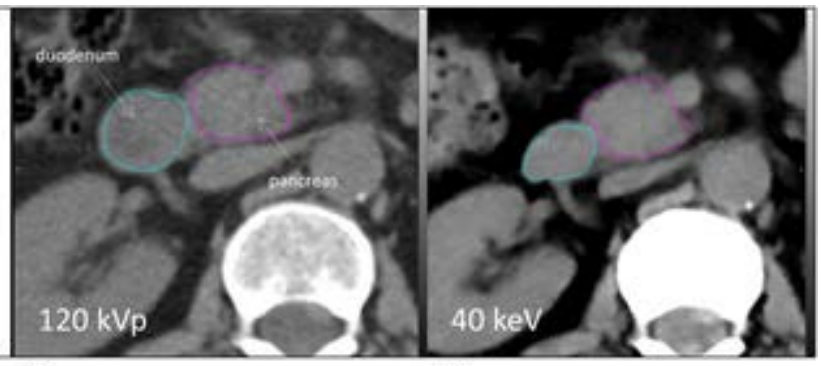

(c)

Figure 1 (a) The contrast (difference in mean CTN) between the pancreas and duodenum for the 10 patients in the study measured at $120 \mathrm{kVp}$ and $40 \mathrm{keV}$. (b) An axial slice of one of the patients scanned at $120 \mathrm{kVP}$. The reconstruction kernel is $30 \mathrm{f}$, the slice thickness is $3.0 \mathrm{~mm}$, the pitch is 0.6 , and the CTDIvol was $17 \mathrm{mGy}$. (c) An axial slice of the same patient scanned with the Dual Spiral Dual Energy. The first scan is at $80 \mathrm{kVp}$ with $3.0 \mathrm{~mm}$ slice thickness and a pitch of 0.6. The second scan is at $140 \mathrm{kVp}$ with $3.0 \mathrm{~mm}$ slice thickness and a 1.2 pitch. The reconstruction kernel was 30f. The summed CTDIvol for both scans was $22 \mathrm{mGy}$. The W/L is 400/40 HU for (b) and (c). [Color figure can be viewed at wileyonlinelibrary.com] 
For one of the selected patients [\#5 in Fig. 1 $1(\mathrm{a})$ ], a 40-keV MEI axial slice from the first week (a) and last week (b) are shown at the top of Fig. 2. The bottom two panels display $120 \mathrm{kVp} \mathrm{PEI}$ axial slices from the treatment days immediately following the DECT scans (c and d). The magenta contour is the pancreatic head (the target) while the light blue contour is the central $8 \mathrm{~mm}$ of the aorta. From the first week to the last week of treatment, the MCTN in the pancreatic head dropped from 23.1 to $18.1 \mathrm{HU}$ as measured in the $120 \mathrm{kVp}$ PEls, a $\triangle C T N$ of $4.9 \mathrm{HU}$. However, for the same time period in the $40 \mathrm{keV} \mathrm{MEI}$, the MCTN dropped from 39.8 to $24.5 \mathrm{HU}$, a $\triangle \mathrm{CTN}$ of 15.3 $\mathrm{HU}$. The CTN variance in the pancreatic head is of the order of $30 \mathrm{HU}$ in a $120 \mathrm{kVp}$ PEI and $50 \mathrm{HU}$ in a $40 \mathrm{keV} \mathrm{MEI}$ which is a reflection of the heterogeneity of pancreatic tissue. However, the mean value is quite stable. For instance, in the aorta, outside of the radiation field, the MCTN only dropped by $0.1 \mathrm{HU}$ in the $120 \mathrm{kVp}$ images and $1.0 \mathrm{HU}$ in the $40 \mathrm{keV}$ images.

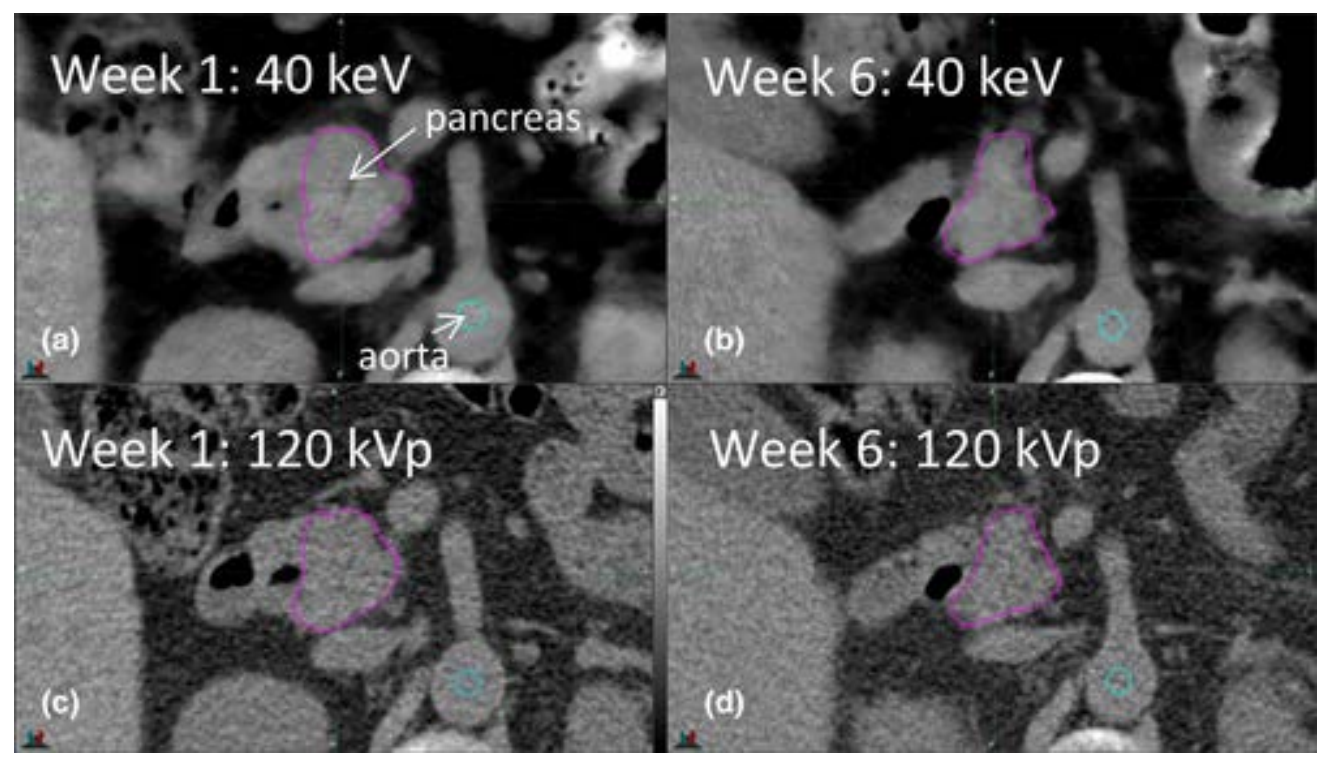

Figure 2 (a) and (b) Axial slices of a patient scanned with the Dual Spiral Dual Energy protocol during the first and last week of treatment. The first scan is at $80 \mathrm{kVp}$ with $3.0 \mathrm{~mm}$ slice thickness and a pitch of 0.6. The second scan is at $140 \mathrm{kVp}$ with $3.0 \mathrm{~mm}$ slice thickness and a $1.2 \mathrm{pitch}$. The reconstruction kernel is $30 \mathrm{f}$. The summed CTDIvol for both scans is $22 \mathrm{mGy}$. The pancreas head is contoured in magenta and the center of the aorta is contoured in light blue. (c) and (d) An axial slice of the same patient as in A\&B scanned at $120 \mathrm{kVp}$. The reconstruction kernel is $30 \mathrm{f}$, slice thickness is $3.0 \mathrm{~mm}$, pitch is 0.6 , and the CTDIvol is $18 \mathrm{mGy}$. The $\mathrm{W} / \mathrm{L}$ is $400 / 40 \mathrm{HU}$ for (a-d). [Color figure can be viewed at wileyonlinelibrary.com]

A bar graph of the change in mean CTN in the target of all the patients is shown in Fig. $\underline{3}(\mathrm{a})$ along with the details of each case in Fig. $\underline{3}(b)$. For all cases we examined the magnitude of the change in mean CTN as measured in the $40 \mathrm{keV} \mathrm{MEI}$ in comparison to the $120 \mathrm{kVp}$ PEI. The average change in mean CTN was $-11.1 \mathrm{HU}$ for MEI, whereas for PEI it was $-4.1 \mathrm{HU}$. In the aorta, the mean CTN was stable; the average changes in the MEI and PEI were $-1.0 \mathrm{HU}$ and $-0.4 \mathrm{HU}$, respectively. Note that for one of the patients, the change in mean CTN in the pancreas head is comparable to the change in the mean CTN outside of the treatment field (patient 4 in Fig. $\underline{3}$ ) independent of whether the measurement is done with PEI or MEI. For this patient the pathological response was graded a 2 (or partial response) on the Ryan scheme for tumor regression. Hence, MEl did not create a CTbased treatment response signal that was not evident in the PEI which is an essential feature of the treatment response enhancement. 


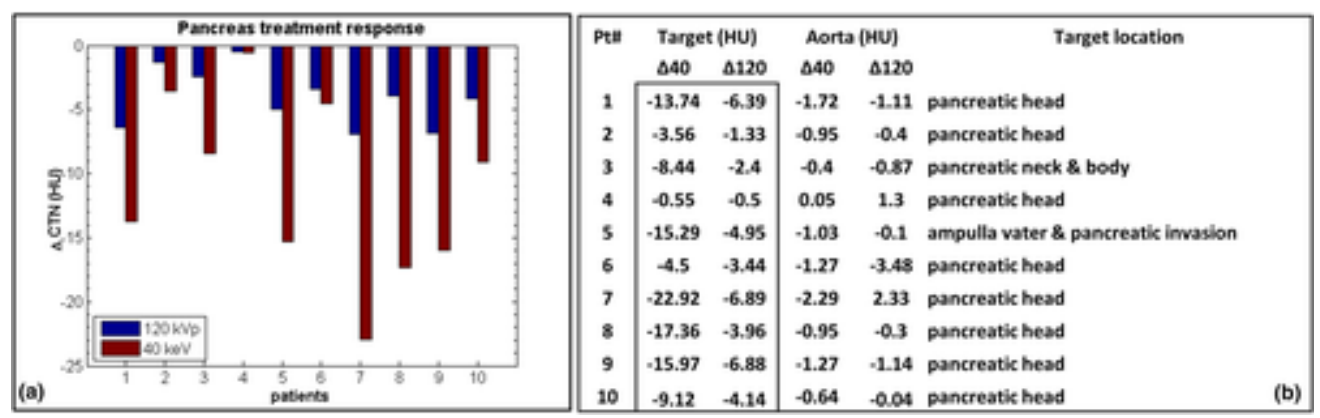

Figure 3 (a) The change in the mean CTN between the first and last week of treatment for the 10 pancreas patients in the study as measured at $120 \mathrm{kVp}$ and $40 \mathrm{keV}$. (b) A table summarizing the mean CTN change (HU) in the target and aorta along with the target location for each patient. [Color figure can be viewed at wileyonlinelibrary.com]

Figure $\underline{4}$ compares the changes in selected histogram features (mean CTN and normalized entropy) during treatment for two patients between $120 \mathrm{kVp} \mathrm{PEI}$ and $40 \mathrm{keV} \mathrm{MEI.} \mathrm{The} \mathrm{radiation-induced} \mathrm{changes} \mathrm{in} \mathrm{CT} \mathrm{textures,}$ both magnitude and timing, are patient specific as indicated for the two patients in Fig. $\underline{4}$. For instance, the changes in mean CTN for patients 7 and 8 are of the same order of magnitude, but for patient 7 the change is roughly linear vs time, while for patient 8 the change occurs during a single week. Figure $\underline{5}$ is a plot of the change in selected CT histogram features of the pancreas head for patient 7 as a function of treatment week. The five features (skewness, kurtosis, entropy, standard deviation, and mean CTN) are normalized independently to their values obtained from the pancreas head in the scan from the first week for $40 \mathrm{keV} \mathrm{MEI} \mathrm{and} 120 \mathrm{kVp}$ study sets. Note that the change in entropy was the largest relative change among the selected histogram features. Figure $\underline{6}$ compares the changes in entropy from the first week to last week of treatment for the 10 patients between data from $40 \mathrm{keV} \mathrm{MEI}$ and $120 \mathrm{kVp}$ PEl.
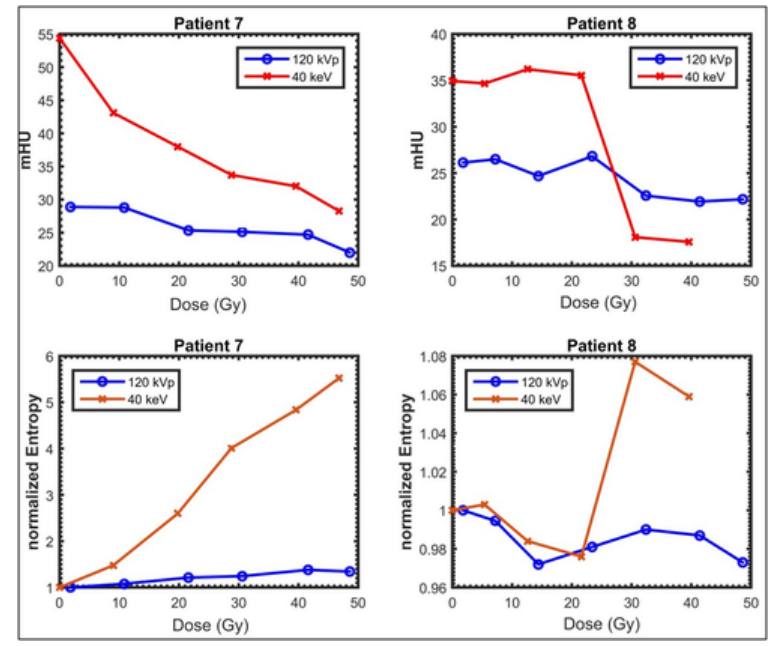

Figure 4 Changes in selected histogram features (mean CTN and normalized entropy) for two patients during treatment for both $120 \mathrm{kVp} \mathrm{PEI}$ and $40 \mathrm{keV} \mathrm{MEI.} \mathrm{[Color} \mathrm{figure} \mathrm{can} \mathrm{be} \mathrm{viewed} \mathrm{at} \mathrm{wileyonlinelibrary.com]}$ 


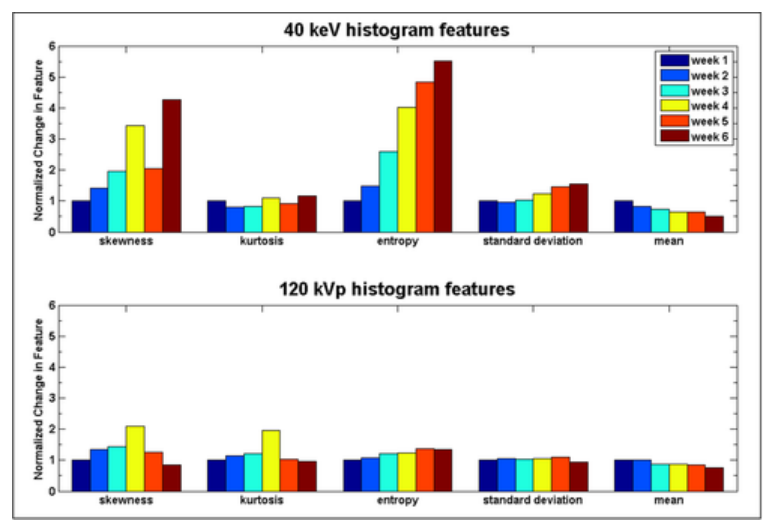

Figure 5 The relative change in five first-order texture features for pt 7 from $40 \mathrm{keV} \mathrm{MEI} \mathrm{and} 120 \mathrm{kVp} \mathrm{PEI} \mathrm{as} \mathrm{a}$ function of treatment week. [Color figure can be viewed at wileyonlinelibrary.com]

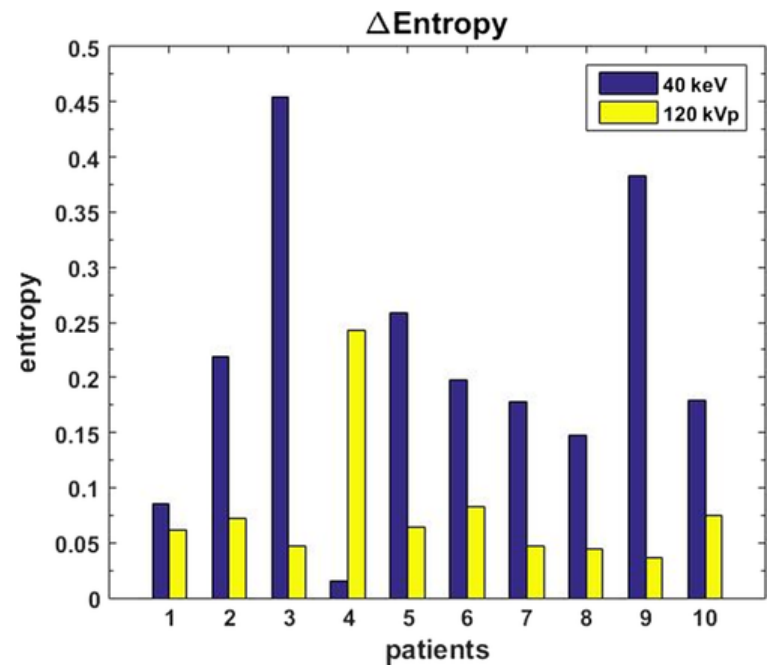

Figure 6 The change in histogram entropy for the 10 patients in the study from $40 \mathrm{keV}$ MEl and $120 \mathrm{kVp} \mathrm{PEI}$ as a function of treatment week. [Color figure can be viewed at wileyonlinelibrary.com]

\section{Discussion}

This study had two aims. First it sought to quantify the CT contrast enhancement available through the use of DECT for pancreas cancer. Specifically, low-energy MEI generated from a sequential DECT protocol feature substantially increased soft tissue contrast relative to traditional PEls. For radiation oncology applications such as IGRT, relatively poor soft tissue contrast of conventional CT can lead to uncertainty in soft tissue-based registration. Improved image quality results in reduced uncertainty in soft tissue registration as described by Morrow et al.13 CT-based RT planning also depends on adequate soft tissue contrast to accurately delineate the edge between targets and organs at risk. Secondly, it sought to measure the amplification of the CT texturebased treatment response signal during pancreatic cancer RT from low-energy MEI relative to traditional PEI. As reported previously, the radiation-induced CT texture changes during chemo-RT for pancreatic cancer correlated with tumor pathological response, although the changes were generally small from the conventional $120 \mathrm{kVp}$ CTs.14 We seek to demonstrate that low-energy MEI will increase the magnitude of the signal and thus make the radiation-induced changes in $\mathrm{CT}$ textures easier to detect during fractionated treatment. The determination of a threshold in the signal that would motivate an adaptive therapy plan is beyond the scope of this exploratory study but is a goal of future work. 
The sequential MEI protocol used in this study does have limitations. Patient motion between the two acquisitions of the sequential protocol can result in artifacts in the MEl. Furthermore, the Dual Spiral Dual Energy sequential protocol was not compatible with respiratory-gated CT scans. All the pancreatic patients in this study had a tumor respiratory motion smaller than $7 \mathrm{~mm}$ as measured by 4DCT acquired at the time of simulation. For patients with larger tumor respiratory motion, daily CT and treatment were gated in our institution which precluded them from the benefit of the sequential DECT protocol. It is our finding that roughly one-third of our pancreas cancer patients require respiratory gating. There are typically two pancreatic cancer patients treated on the linac with the DECT protocol at any given time in our clinic.

Dual Spiral Dual Energy protocol is designed to be dose neutral compared to the standard protocol $120 \mathrm{kVp}$ for a given patient. Radiation therapy patients, being treated for tumors in the pancreas head received doses of 50$70 \mathrm{~Gy}$. The patients received CT guidance at each fraction to assure their positioning accuracy. As a result, they received an additional dose of radiation that increased their overall dose by roughly $0.5 \mathrm{~Gy}$ (18 mGy in 28 fractions), or less than $1 \%$ of their total dose. If one considers the integrated dose, our analysis indicates that the IGRT dose is closer to $10 \%$ of the therapeutic dose; $\mathrm{kV}$ cone-beam IGRT scans, which are more common, require as much as $50 \%$ more dose than the CT-on-rails imaging used here. DECT protocols may expose the patients to slightly elevated doses if their body width was larger than average. However, when these protocols are coupled with dose reduction algorithms such as CARE dose 4D, the benefits of DECT are achievable with a dose increase no more than $4 \mathrm{mGy}$ per fraction employed. CARE dose 4D modulates the dose based on the patient width as measured from the topogram. Larger than average patients will receive a slightly elevated dose so that noise is reduced to a level that would be similar to patients of average diameter. Patients with smaller than average abdomen diameters had their dose reduced due to the application of the dose reduction algorithm. $1 \mathbf{8}$

For pancreatic cancer, treatment response was difficult to detect with standard CT because of the poor soft tissue contrast. Soft tissue features of the glands and embedded tumor were nearly indistinguishable in $120 \mathrm{kVp}$ PEls [see Fig. $\underline{1}$ (b) and Figs. $\underline{2}$ (c) and $\underline{\mathbf{2}}$ (d)]. These images depended on a spectrum of x-ray energies which have different scattering properties. Low-energy MEI such as those generated from dual-energy protocols benefitted from the photoelectric effect, resulting in a much broader range of CT numbers. As the x-ray energy is decreased, the contrast in the resulting images rapidly increased, enabling a differentiation between soft tissue types. Enhancing the contrast between soft tissues in a single scan, as demonstrated by Fig. 1, can also amplify the detection of treatment response as measured from successive scans over the course of fractionated treatment.

The RT-related changes in the composition of the tumor and surrounding tissue are amplified as demonstrated by increased changes in the MEI target textures relative to the same textural changes in PEI (see Fig. $\underline{5}$ ). Histogram features can vary during fractionated treatment and have been correlated with histological outcome for pancreas cancer patients.14 The amplification of this change for a single patient via low-energy MEl is immediately evident in Fig. $\underline{5}$, but correlating that to treatment response is beyond the scope of this study. Weekly variation in a single histogram feature for a single patient may or may not be related to treatment response, but our analysis demonstrates that the MEI of DECT augments those changes. Therefore, treatment response would be easier to detect via the MEI of DECT.

Periodic DECT during fractionated treatment may enable the detection of response earlier than was previously achievable from standard CT. Specifically for pancreas cancers, the response signal may be difficult to distinguish from noise after the first few weeks of treatment using standard CT. The change in PEI CT intensity after completing therapy is only expected to be on the order of $5 \mathrm{HU}$ for pancreatic tumors.14 During early fractions, the change would be expected to be even less than that, and thus may be inadequate to motivate adaptive planning. By periodically scanning a patient with a DECT protocol, the clinician can expect a much larger treatment response signal in the MEI (see Figs. $\underline{4}$ and $\underline{5}$ ). For the 10 patients in our study the average reduction 
in $\mathrm{CT}$ number was $1 \mathrm{HU}$ in the $40 \mathrm{keV} \mathrm{MEI}$ and $4 \mathrm{HU}$ in the $120 \mathrm{kVp} \mathrm{PEI}$. As a consequence, adaptive replanning would be indicated sooner for appropriate patients.

\section{Conclusions}

Radiation therapy planning and delivery can be improved by using DECT. Low-energy monoenergetic images from DECT can enhance soft tissue contrast relative to standard CT as demonstrated in this study. For all patient data analyzed, DECT substantially increased the difference in CTN between the pancreas head and duodenum. Furthermore, MEI/DECT amplified the changes in CT textures during radiation delivery, potentially allowing an earlier detection of treatment response. The use of monoenergetic $40 \mathrm{keV} \mathrm{MEI}$ from DECT enhanced the radiation-induced change in the mean CTN in the target by a factor of 2-3 compared to what was measured with standard CT for the pancreatic cancer patients studied. The amplifications of entropy from MEI vs PEI are up to a factor of 5 for the selected patients.

\section{Acknowledgment}

This work is partially supported by Siemens Medical Solutions USA, Inc. and by the MCWCC Fotsch Foundation.

\section{References}

1Johnson TR. Dual-energy CT: general principles. Am J Roentgenol. 2012; 199: S3- S8.

2Alvarez RE, Macovski A. Energy-selective reconstructions in X-ray computerized tomography. Phys Med Biol. 1976; 21: 733- 744.

3Yu L, Leng S, McCollough C. Dual-energy CT-based monochromatic imaging. Am J Roentgenol. 2012; 199: S9-S15.

4Watanabe Y. Derivation of linear attenuation coefficients from CT numbers for low-energy photons. Phys Med Biol. 1999; 44: 2201- 2211.

5Kretschmer M, Sabatino M, Heyden S, Loeffler W, Wagner M. Dual Energy CT for Metal Artifact Reduction in Radiation Therapy Planning with the SOMATOM Definition AS 20. Siemens Medical Solutions Whitepaper. 2013.

6Schneider D, Apfaltrer $\mathrm{P}$, Sudarski S, et al. Optimization of kiloelectron volt settings in cerebral and cervical dual-energy $\mathrm{CT}$ angiography determined with virtual monoenergetic imaging. Acad Radiol. 2014; 21: 431- 436.

7Wichmann J, Noske EM, Kraft J, et al. Virtual monoenergetic dual-energy computed tomography: optimization of kiloelectron volt settings in head and neck cancer. Invest Radiol. 2014; 49: 735- 741.

8Sudarski S, Apfaltrer P, Nance JW, et al. Optimization of keV-settings in abdominal and lower extremity dualsource dual energy CT angiography determined with virtual monoenergetic imaging. Eur J Radiol. 2013; 82: e574- e581.

9Sudarski S, Apfaltrer P, Nance JW, et al. Objective and subjective image quality of liver parenchyma and hepatic metastases with virtual monoenergetic dual-source dual-energy CT reconstructions: an analysis in patients with gastrointestinal stromal tumor. Acad Radiol. 2014; 21: 514- 522.

10Kaup M, Scholtz JE, Engler A, et al. Dual-energy computed tomography virtual monoenergetic imaging of lung cancer: assessment of optimal energy levels. J Comput Assist Tomogr. 2016; 40: 80-85.

11Frellessen C, Fessler F, Hardie AD, et al. Dual-energy CT of the pancreas: improved carcinoma-to-pancreas contrast with a noise-optimized monoenergetic reconstruction algorithm. Eur J

Radiol. 2015; 84: 2052- 2058.

12 Hardie D, Picard MM, Camp ER, et al. Application of an advanced image-based virtual monoenergetic reconstruction of dual source dual-energy CT data at low keV increases image quality for routine pancreas imaging. J Comput Assist Tomogr. 2015; 39: 716- 720. 
13Morrow N, Lawton CA, Qi XS, Li XA. Impact of computed tomography image quality on image-guided radiation therapy based on soft tissue registration. Int J Radiat Oncol Biol Phys. 2012; 82: e733-e738.

14Chen X, Oshima K, Schott D, et al. Assessment of treatment response during chemoradiation therapy for pancreatic cancer based on quantitative radiomic analysis of daily CTs: an exploratory study. PLoS ONE. 2017; 12: e0178961.

15Feng $\mathrm{M}$, Yang $\mathrm{C}$, Chen $\mathrm{X}$, et al. Computed tomography number changes observed during computed tomography-guided radiation therapy for head and neck cancer. Int J Radiat Oncol Biol Phys. 2015; 91: 1041- 1047.

16Mayer R, Stanton K, Kleinberg L, Charkravarthy A, Fishman E. CT number distribution and its association with local control and as a marker of lung tumor response to radiation. Radiat Oncol Invest. 1998; 6: 281- 288.

17Herfarth K, Hof H, Bahner ML, et al. Assessment of focal liver reaction by multiphasic CT after stereotactic single-dose radiotherapy of liver tumors. Int J Radiat Oncol Biol Phys. 2003; 57: 444- 451.

18Kalra MK, Maher MM, Toth TL, et al. Techniques and application of automatic tube current. Radiology. 2004; 233: 649- 657.

19Grant K, Raupach R. SAFIRE: Sinogram Affirmed Iterative Reconstruction. Siemens Medical Solutions Whitepaper A911IM-CT-121036-P1-4A00; 2012.

20Noid G, Tai $A$, Chen GP, et al. Reducing radiation dose and enhancing imaging quality of 4DCT for radiation therapy using iterative reconstruction algorithms. Adv Radiat Oncol. 2017; 2: 515- 521. 